\title{
L-carnosine as an enhancer to computerized cognitive behaviour therapy in Japanese workers, an unjustified claim
}

\author{
Geneviève Sauvé, Marie-France Bastien, Casandra Roy-Gelencser and Ghassan El-Baalbaki ${ }^{*}$
}

\begin{abstract}
This letter comments on the conclusion drawn by Shirotsuki et al. (2017) in their article entitled "The effect for Japanese workers of a self-help computerized cognitive behaviour therapy program with a supplement soft drink", recently published in BioPsychoSocial Medicine. The authors concluded that their drink, containing L-carnosine, enhances the effects of a computerized cognitive-behavioural therapy (CCBT) on the psychological well-being of healthy Japanese workers. Yet, we argue that their conclusion is unfounded given their results and the methodological shortcomings of their study. Briefly, while the authors reported improvement on the tensionanxiety subscale of the Profile of Mood States (POMS) in the CCBT only group, they also observed a lack of improvement on this subscale in the CCBT+L-carnosine group suggesting that the drink washes out this beneficial effect of CCBT. Methodological issues include the uncontrolled levels of L-carnosine metabolized by participants jeopardize the study's internal validity. Also, the clinical meaningfulness of the findings seems dubious as post-treatment scores remained within the range of the general Japanese population. Consequently, we argue that Shirotsuki et al.'s study should be re-conducted before drawing any valid conclusion.
\end{abstract}

Keywords: Psychological well-being, Fatigue, Anxiety, Supplement, L-carnosine, Computer-based cognitivebehavioural therapy (CCBT), Clinical significance

Shirotsuki et al.'s study [1] assessed whether the intake of a carbonated drink containing L-carnosine increases the effects of a computerized cognitive-behavioural therapy (CCBT) on psychological well-being of healthy Japanese workers. To achieve their goal, the authors conducted a randomized-controlled trial with three groups: an experimental group $(n=29)$ receiving the dietary supplement in combination with the CCBT, a first control group $(n=29)$ receiving only CCBT, and a second control group $(\mathrm{n}=29)$ receiving neither of the two forms of intervention. The CCBT used in this study is a six-week psychoeducational personal development program addressing stress management and coping via behavioral activation and cognitive restructuring. The dietary supplement used in this study, the L-carnosine molecule, is a widely studied dipeptide with antioxidant properties. Participants of the study consumed $200 \mathrm{mg}$ of L-carnosine in a $100 \mathrm{ml}$ soft drink each morning during the 6 weeks of intervention. Psychological well-being was operationalized via measures of anxiety, depression and fatigue levels. Additional measures of self-efficacy as well as medical and psychosomatic symptoms were also reported. Following their analyses, the authors concluded that consuming a soft drink containing $200 \mathrm{mg}$ of L-carnosine increased the effectiveness of the CCBT on fatigue levels. However, we believe that the authors' conclusion is unfounded given their

\footnotetext{
* Correspondence: el-baalbaki.ghassan@uqam.ca

Département de psychologie, Université du Québec à Montréal, C.P. 8888

succursale Centre-ville, Montréal, Québec H3C 3P8, Canada
}

(c) The Author(s). 2018 Open Access This article is distributed under the terms of the Creative Commons Attribution 4.0 International License (http://creativecommons.org/licenses/by/4.0/), which permits unrestricted use, distribution, and reproduction in any medium, provided you give appropriate credit to the original author(s) and the source, provide a link to the Creative Commons license, and indicate if changes were made. The Creative Commons Public Domain Dedication waiver (http://creativecommons.org/publicdomain/zero/1.0/) applies to the data made available in this article, unless otherwise stated. 
results and the methodological shortcomings of the study that we will detail below.

Beforehand, we wish to acknowledge the authors' transparency regarding the involvement of Suntory Global Innovation Center Limited in the study's funding and execution, as well as the multiple roles held by two of their employees as authors ( $\mathrm{YN}$ and $\mathrm{KA}$ ) and creators of the drink containing L-carnosine. In the study, it was also reported that participants were employees of a Suntory Group's production factory. We thought important to remind the reader about the context in which this experimental research took place, given the high threat to internal validity caused by the well documented investigators' bias as well as the subjects' and participation biases [2-4], making the quality of this study low as per the Cochrane Risk of Bias Tool for Randomized Controlled Trials ([5], Table 8.5.d). More precisely, in Shirotsuki et al.'s study [1], we found a high risk of bias in selective reporting, incomplete outcome data, and blinding of participants and outcome assessment, meaning that conclusions of such low quality studies must be cautiously taken.

Our first methodological question, refers to the uncontrolled consumption of L-carnosine by participants in their regular diet. The verification of L-carnosine can be made using High Performance Liquid Chromatography (HPLC) on urine or plasma samples [6]. The lack of doing so causes a threat to the internal validity: the improvement observed in the L-carnosine group on the POMS-fatigue subscale could be explained by the participants' regular diet and not the drink. As indicated by the authors, significant amounts of L-carnosine are present in meat (e.g., poultry and beef), which can promote recovery following a state of mental fatigue. Alternatively, 1participants' diet can be assessed longitudinally using a food-intake journal, 2- they can be asked to maintain their dietary habits throughout the study, and/or 3- background diet (e.g., vegetarianism, dietary supplements intake) can be used as an exclusion criterion as suggested by Woodside, et al. [7].

Another question we have for the authors, has to do with the clinical significance associated with the reported statistically significant results. How come the authors did not calculate a reliable change index, classically proposed by Truax and Jacobson in 1991 and Speer in 1992 [8], in order to grant that these statistically significant changes reflect a clinically meaningful improvement and not only a statistical regression towards the mean. A number of external factors could therefore explain the observed group differences. Moreover, the post-treatment means on the 'tension-anxiety' and the 'fatigue' POMS subscales, remain within the range of scores exhibited by the general Japanese population (mean \pm 1SD) [9]. Hence, the statistical improvement reported, could be the result of participant's bias (acquiescence response style) [2]. Further, the psychometric validation study of the Japanese version of the POMS used a sample constituted only of men and did not report any clinical threshold or cutoffs [10]. Consequently, without a clear indication that the gains observed following the intervention had a noticeable clinical impact in participants' lives [2], it becomes problematic to claim that CCBT improves the subjective perception of well-being and that L-carnosine increases such effects. Which, again calls for the calculation of a clinically reliable index. It is safe to conclude that implementing such a program is yet unwarranted, given that the post-treatment scores do not distinguish the trial participants from what is observed in the general Japanese population [9].

Finally our last remark, is about the lack of improvement on the tension-anxiety subscale in the combined group (CCBT+L-carnosine), whereas the CCBT group without drink, had shown improvement $($ Cohen's d effect size $=.37)([1]$, p.5). At first glance if we dismiss all the methodological issues raised that could render this result non valid - we could conclude that adding a L-carnosine drink to CCBT is deleterious to CCBT for stress management! In other words, the addition of the authors' mother industry drink to CCBT, reduces fatigue but washes-out the beneficial effect of CCBT on tension-anxiety. Tension and anxiety are correlated to stress, and tension, anxiety and stress are correlated to fatigue. So here are some additional questions: 1- authors reported that a measure of treatment adherence was completed but no results were given. How did the participants of the CCBT+drink compare to the CCBT only protocol? 2- if tension-anxiety is not enhanced, how long the fatigue improvement will last, given that prolonged presence of tension and anxiety leads ultimately to fatigue. 3- What is the rationale of a stress management treatment that does not address all main symptoms and causes?

In conclusion, we wish to acknowledge that Shirotsuki, et al. [1] reported the limitations of their study (e.g., absence of a control group with a placebo drink, absence of a control group with the L-carnosine drink only, social desirability of participants). Nevertheless, these limits remain highly threatening to the internal validity and our questions relative to what looks like serious methodological shortcomings should lead to only one conclusion: the study of Shirotsuki, et al. [1] should be re-conducted before any valid conclusion could be drawn. 


\author{
Reply to the comment on L-carnosine as an enhancer of computerized cognitive behaviour therapy in \\ Japanese workers \\ Kentaro Shirotsuki ${ }^{1}$, Yuji Nonaka ${ }^{2}$, Keiichi Abe ${ }^{3}$, So-ichiro Adachi ${ }^{4}$, Shohei Adachi ${ }^{4}$, Tomifusa Kuboki ${ }^{5}$ and \\ Mutsuhiro Nakao ${ }^{6}$ \\ ${ }^{1}$ Faculty of Human Sciences, Musashino University, Tokyo, Japan \\ ${ }^{2}$ Innovation Development Department, Suntory Global Innovation Center Limited, Osaka, Japan \\ ${ }^{3}$ National Institute of Health and Nutrition, Tokyo, Japan \\ ${ }^{4}$ Medical corporation So-bun-kai, Clinic Adachi, Gifu, Japan \\ ${ }^{5}$ Professor Emeritus, Tokyo University, Tokyo, Japan \\ ${ }^{6}$ Faculty of Medicine, International University of Health and Welfare, Tokyo, Japan
}

Abstract

This article responses to the comments on the study entitled "The effect for Japanese workers of a self-help computerized cognitive behavior therapy program with a supplement soft drink" [1]. Certain limitations of the study, such as participants, measurements, and the study protocols among others have been pointed out in these comments. However, the possible limitations of the research were explicitly addressed in the original article [1] and future tasks were discussed before making any conclusions. It was suggested that future studies should use a larger sample of participants from independent institutions and communities to confirm our results. We also strictly evaluated compliance by conducting the program as objectively as possible after randomization. However, more efforts are needed to implement more rigorous designs in the future. Moreover, because many factors are known to differentially affect each mood state, we individually discussed mechanisms that decrease anxiety and fatigue. The strength of the study is that all the procedures were described to facilitate re-testing by other investigators and the results of all the measured variables were shown, regardless of whether they were statistically significant or not. It is suggested that more investigators should be involved in examining additional effects of supplements on cognitive behavioral intervention.

Keywords: Computerized cognitive behaviour therapy, Workplace, L-carnosine, Self-help

In the original study [1] we examined whether a supplement drink that includes L-carnosine enhances the effect of CCBT on psychological well-being. The results suggested that the self-help in the CCBT program reduced the subjective experience of tension-anxiety in a group of workers. The addition of a supplement drink enhanced the effect of CCBT on fatigue. Therefore the CCBT program and the addition of a supplement drink was considered one possible approach to enhancing the effects of CCBT programs on anxiety and fatigue. An article commenting on the study has pointed out certain problems and limitations of this research. However before marking the final conclusions of the original study, we described the same limitations and explicitly addressed them

\section{Participants}

The Suntory Global Innovation Center Limited supported this study and two of their employees were the authors (YN and KA) and the developers of the drink containing L-carnosine. We also reported that study-participants were employed in a Suntory Group factory. We pointed out in the section on the limitations of the study in the original article that the support given by the Suntory group for this study and having Suntory employees as participants was a limitation of the study and that there was room for improving the study. Nevertheless, employees of the division responsible for this study did not participate in the study. Additionally, a simple randomization procedure was conducted by an independent study controller who was not an employee of the Suntory Global Innovation Center Limited and had no direct contact with the participants. Therefore, the issue concerning the selection of participants, we took all possible measures to ensure the reliability of the study.

Moreover, there was no arbitrariness in reporting the study results because we reported all the results including those that were not statistically significant. Furthermore, an independent third party was in charge of data analysis and missing values among others were properly processed. Also, all the indications suggest that the data were complete. Furthermore, because this research involved a company, we clearly stated all the necessary information including the COI disclosure. However, blind participation procedures are impossible in CBT studies. As a result, we did not consider using blinding and 
randomizing procedure when drinking the beverage because the main objective of this study was to test the effects of CBT. Therefore, it is suggested that a large-scale study be undertaken in the future with participants from outside the Suntory corporation and also include a procedure for blindly drinking the beverage.

\section{Consumption of L-carnosine and daily dietary records}

As indicated in the comments, it was important to correctly check the participants' regular diet and eating habits. Therefore, in the study, we also asked the participants to regulate their diet and eating habits at the time of requesting their consent to maintain lifestyle habits and refrain from overeating and overdrinking during the program. Moreover, we analyzed the data of participants with a supplement drink intake rate higher than $90 \%$. These procedures were similar to previous works [11-13]. In this study, we also conducted randomization to ensure that dietary habits of the three groups were identical. In addition to teaching the development of lifestyle habits as a part of the CCBT program, we also recorded the meals of the control group and conducted minimal management. The study by Shirotsuki et al. [1] was done mainly to conduct and assess the CCBT program. In the study, the participants recorded data on their daily life activities, and the programs that they followed on worksheets. We did not ask the participants to strictly control and record their diet because it might have placed a heavy burden on the participants and resulted in dropouts and noncompliance. Moreover, the main assessments of the study were psychological indices and no physiological indices were used. It is suggested that future studies should assess physiological indices to clarify overall changes.

The measurement of physiological changes is a necessary task for the future. However, in the study by Shirotsuki et al. [1] the participants experienced a minimal degree of control. Also, excessive recording and management might have decreased the motivation and increased the drop-out rate from the CCBT program. Therefore, in this study, we did not require that participants perform too many tasks or recordings to ensure their compliance.

\section{POMS}

It is true that the cited article on POMS [14] is based only on male participants. However, the Japanese version of POMS has been analyzed with an enormous amount of data of healthy and clinical samples of men and women, and the scores have been standardized. POMS is widely recognized as useful in Japan, and it has well demonstrated clinical significance [14]. Several BioPsychosocial Medicine studies [15-17] have also used POMS. In recent years, POMS-2 has been developed and refined. POMS has been properly standardized, but additional refinements including measurements on different scales could be useful.

The study by Shirotsuki et al. [1] was a pilot study conducted with healthy participants to assess psychological traits of the participants. In the study, participants with mental or physical health risks and those with high POMS scores were excluded. It would be necessary to investigate participants with psychological or mental health problems to establish the clinical significance of the study. Nevertheless, Shirotsuki et al. suggested primary preventive measures based on the investigation of healthy participants.

\section{Compliance and anxiety and fatigue}

To assess the compliance with the program, participants with a drink intake rate of less than $90 \%$, those that maintained a life record less than $90 \%$ of the time, those that conducted the CBT task less than $90 \%$ of the time, those that did not complete watching the CBT videos six times, and those showing considerably large variations in their life records of sleep among others were excluded from the analysis. The subjects of analysis were participants with a high performance who satisfied these conditions. Therefore, the compliance of the subjects in this study was high.

Although fatigue and anxiety might be related, there are many factors that affect fatigue. Fatigue and anxiety might not change in the same way. There is little clear evidence that anxiety and fatigue in this study were closely related. From supplementary data of the correlation between TA and F in the CCBT + Drink group, it is difficult to conclude that $r=.28$ indicate a strong relationship with each other. Supplementary reporting, in the CCBT + Drink group, the effect size of POMS-TA was as low as $d=.19$. The comments suggested the possibility that the supplement drink might inhibit the reduction of anxiety. However, based on the data above, we do not agree with this suggestion. Moreover, the CCBT program conducted in this study was a Self-Help program. Participants themselves could have dealt with the part of the program related to stress management. Moreover, the program was rather simple, and it was possible to complete it in only six sessions. Therefore, its effect might not have been significant. The results might be improved by increasing the number of sessions and support provided by therapists, which would make the findings of this study more robust. Generally, CCBT programs are more effective for anxiety and depression, rather than for physical symptoms, which might have affected the results of this study. 


\section{Conclusion}

There are limitations to this study, and future studies are needed to clarify specific issues. Nevertheless, this study suggested the possible positive effects of simultaneously using a CCBT program and L-carnosine. Future studies could improve the design of this study by including a placebo drink group. Moreover, using a different research design could improve the CCBT program. Furthermore, different researchers should conduct this investigation in the future with different participants. Shirotsuki et al. [1] used randomization procedures; however, there were between-group differences in specific indices. Therefore, it might be possible to obtain clearer results by developing research designs that eliminate between-group disparities.

\section{Abbreviations}

CCBT: Computerized cognitive-behavioral therapy; POMS: Profile of Mood States; HPLC: High Performance Liquid Chromatography; POMS-TA: POMS tension/anxiety subscale; POMS-F: POMS fatigue subscale

\section{Acknowledgements}

We wish to thank Ms. Sachiyo Kanzaki for her help with the translation of Japanese articles.

\section{Funding}

Non-applicable.

\section{Authors' contributions}

All the authors contributed equally, and read and approved the final version.

\section{Ethics approval and consent to participate}

Non-applicable.

\section{Consent for publication}

Non-applicable.

\section{Competing interests}

The authors declare that they have no competing interests.

\section{Publisher's Note}

Springer Nature remains neutral with regard to jurisdictional claims in published maps and institutional affiliations.

\section{Received: 27 April 2018 Accepted: 6 August 2018}

Published online: 04 September 2018

\section{References}

1. Shirotsuki K, Nonaka Y, Abe K, Adachi SI, Adachi S, Kuboki T, Nakao M. The effect for Japanese workers of a self-help computerized cognitive behaviour therapy program with a supplement soft drink. Biopsychosoc Med. 2017;11:23.

2. Kazdin AE. Research design in clinical psychology. Boston: Pearson; 2016

3. Paulhus DL. Two-component models of socially desirable responding. J Pers Soc Psychol. 1984;46:598-609.

4. Keeble C, Barber S, Law GR, Baxter PD. Participation bias assessment in three high-impact journals. SAGE Open. 2013;3(4):1-5.

5. Shuster JJ. Review: Cochrane handbook for systematic reviews for interventions, version 5.1.0, published 3/2011. In: Higgins JPT, Green S, editors. The Cochrane Collaboration, 2011. Available at: www.cochranehand-book.org.

6. Park YJ, Volpe SL, Decker EA. Quantitation of carnosine in humans plasma after dietary consumption of beef. J Agric Food Chem. 2005:53(12):4736-9.

7. Woodside JV, Welch RW, Patterson CC, McKinley MC. Study design: intervention studies. In: Lovegrove JA, Hodson L, Sharma S, L-NS A, editors. Nutrition research methodologies. New Jersey: Wiley; 2015. p. 28-47.
8. Speer DC. Clinically significant change: Jacobson and Truax (1991) revisited. J Consult Clin Psychol. 1992:60(3):402-8.

9. Yokoyama K, Araki S, Okajima F, Nomura S, Okuyama I. Examination of the Japanese edition of profile of mood states (POMS) and short versions. Jpn J Public Health. 1993;40:1055. [in Japanese]

10. Yokoyama K, Araki S, Kawakami N, Kakeshita T. Production of the Japanese edition of profile of mood states (POMS): assessment of reliability and validity. Nippon Koshu Elsei Zasshi. 1990;37:913-8.

11. Tanaka M, Kamohara R, Fujii H, Hirayama Y. Effect of CBEX-Dr-containing Drink on Physical Fatigue in Healthy Volunteers. Jpn Pharmacol Ther. 2008; 36:199-212

12. Shimizu K, Fukuda M, Yamamoto H. Effect of repeated intake of imidazole dipeptides-containing drink on healthy people with feeling of fatigue from daily activities - the results of 207 volunteers enrolled in the $1^{\text {st }}$ recruitment-. Jpn Pharmacol Ther. 2009:37:255-63.

13. Yamano E, Tanaka M, Ishii A, Tsuruoka N, Abe K, Watanabe Y. Effects of chicken essence on recovery from mental fatigue in healthy males. Med Sci Monit. 2013;19:540-7. https://doi.org/10.12659/MSM.883971.

14. Yokoyama K, Shimomitsu T, Nomura S. Shindan Shido ni ikasu POMS jirei syu (in Japanese). Tokyo: Kaneko Syobo; 2002.

15. Oka T, Tanahashi T, Chijiwa T, Lkhagvasuren B, Sudo N, et al. Isometric yoga improves the fatigue and pain of patients with chronic fatigue syndrome who are resistant to conventional therapy: a randomized, controlled trial. Biopsychosoc Med. 2014;8:27. https://doi.org/10.1186/s13030-014-0027-8.

16. Yoshihara K, Hiramoto T, Sudo N, Kubo C. Profile of mood states and stressrelated biochemical indices in long-term yoga practitioners. Biopsychosoc Med. 2011;5(1):6. https://doi.org/10.1186/1751-0759-5-6.

17. Fukuo W, Yoshiuchi K, Takimoto Y, Sakamoto N, Kikuchi H, Hachizuka M, et al. Comparison of temporal changes in psychological distress after hematopoietic stem cell transplantation among the underlying diseases of Japanese adult patients. Biopsychosoc Med. 2008;2:24. https://doi.org/10 1186/1751-0759-2-24.

Ready to submit your research? Choose BMC and benefit from:

- fast, convenient online submission

- thorough peer review by experienced researchers in your field

- rapid publication on acceptance

- support for research data, including large and complex data types

- gold Open Access which fosters wider collaboration and increased citations

- maximum visibility for your research: over $100 \mathrm{M}$ website views per year

At $B M C$, research is always in progress.

Learn more biomedcentral.com/submission 